\title{
Lysophosphatidylcholine in Platelet Microvesicles: The Grease for Cardiovascular Disease
}

\author{
Rory R. Koenen ${ }^{1,20}$ \\ ${ }^{1}$ Department of Biochemistry, Cardiovascular Research Institute \\ Maastricht, Maastricht University, Maastricht, The Netherlands \\ 2 Institute for Cardiovascular Prevention (IPEK), LMU Munich, Munich, \\ Germany
}

Thromb Haemost 2019;119:1202-1204.

In this issue of Thrombosis and Haemostasis, Diehl and Peter demonstrate that microvesicles derived from platelets are enriched in the bioactive lipid lysophosphatidylcholine (LPC), which was shown to activate platelets and which could be measured in unstable atherosclerotic plaques. ${ }^{1}$ Extracellular vesicles (EVs) are small membrane-enclosed cell fragments. Almost every cell type, prokaryotic and eukaryotic, is able to release $\mathrm{EV}$ and this is often intensified after activation or during stress. Although a matter of debate, the current knowledge allows the classification of EV into three main types: (1) the exosomes, which originate from the endosomal compartments of the cell, (2) the microvesicles, which are formed after budding of the outer cell membrane, and (3) the apoptotic bodies that result after the cell undergoes apoptosis. The released EV retains many properties of their parent cells with respect to surface markers and content, providing information about the physiologic state and context of their parent cells or tissue. This makes EV highly interesting for biomedical research, for example, as biomarkers. ${ }^{2}$ Unlike classical biomolecular biomarkers that basically contain information solely about concentration and type, EVs carry information about counts, size, cellular origin, and cargo, to name a couple. It is this high information density that makes EV that attractive. ${ }^{3}$ Regarding cargo, EVs have been found to contain nucleic acids, including micro-ribonucleic acids (RNAs), proteins, and bioactive lipids. During the release process, particular constituents can be enriched in EV. Thus, EV might just function to dispose cellular waste, serve as mediators of intercellular communication, ${ }^{4}$ or both. Needless to say, EVs are not only in sharp focus as novel physiologic players during health and disease, but also as promising biomarkers, not in the last place for cardiovascular disease. $^{5-7}$

Being the first cells known to release EV, platelets are a rich source of EV in circulation. While medical textbooks will mainly discuss their essential functions in hemostasis, platelets also play an important role as immune cells. ${ }^{8}$ Thus, the $\mathrm{EV}$ released by platelets not only enhance fibrin generation ${ }^{9}$

(1D) Rory R. Koenen's ORCID is https://orcid.org/0000-0002-99559730.
Address for correspondence Rory R. Koenen, PhD, CARIM, Maastricht University, P.O. Box 616, 6200 MD Maastricht, The Netherlands (e-mail: r.koenen@maastrichtuniversity.nl).

and thrombosis, ${ }^{10}$ but studies have also shown that they influence the behavior and/or phenotype of endothelial cells, ${ }^{11}$ monocytes, ${ }^{12}$ macrophages,,${ }^{13,14}$ or smooth muscle cells, ${ }^{15}$ often skewing cellular responses toward inflammation. Binding of the EV to the recipient cells, with subsequent internalization preceded the phenotypic shifts in all of the above studies. However, the molecular factors within EV made responsible for causing the effects were different and ranged from cytokines (e.g., CXCL4 $4^{15}$ ) and micro-RNAs (e.g., miRNA $126^{14}$ ) to bioactive lipids (e.g., arachidonic acid ${ }^{11}$ ), reflecting the broad palette of bioactive EV cargo.

In the study by Diehl and Peter, a further active molecular constituent of platelet-derived EV (microvesicles) was identified as LPC by mass spectrometry. Interestingly, the LPC contents in microvesicles were markedly enriched after platelet activation, in particular by thrombin, compared with the LPC contents of the platelets themselves. Also, thrombininduced microvesicles contained more LPC than those from resting platelets. This suggests that LPC is actively formed in or transported into microvesicles after platelet activation. Addition of LPC to platelets resulted in typical responses,for example, integrin activation, exposure of P-selectin, spreading on immobilized fibrinogen, and aggregation, but also less wellestablished effects such as platelet-monocyte aggregate formation and migration toward a gradient of LPC. The authors could explain the response of platelets to LPC by the action of the G2A receptor, not only showing the presence of this receptor in platelets, but also demonstrating a dose-dependent inhibition of the response of platelets to LPC by an antibody against the G2A receptor. To investigate the potential of LPC as a possible biomarker, the authors measured LPC in the blood and arteries of control mice and mice with stable and unstable atherosclerosis. The largest amounts of LPC were found in specimen from mice with unstable plaques. Finally, using state-of-the-art massspectrometry imaging on human vulnerable plaque specimen, the authors observed that LPC accumulated in areas that were considered particularly vulnerable.

The study by Diehl and Peter shows that microvesicles from platelets contain bioactive lipids such as LPC. Further, this LPC

(C) 2019 Georg Thieme Verlag KG Stuttgart · New York
DOI https://doi.org/ 10.1055/s-0039-1693024. ISSN 0340-6245. 


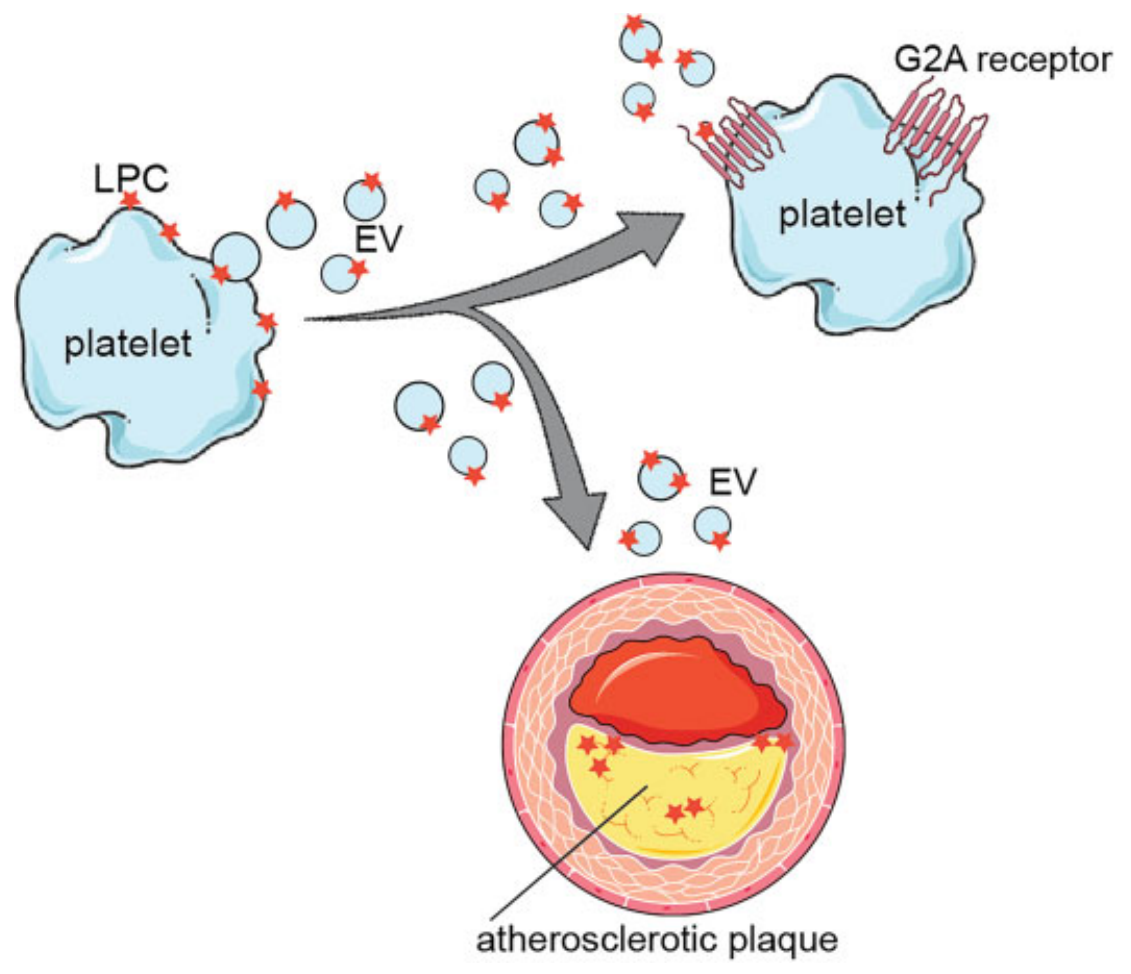

Fig. 1 Lysophosphatidylcholine (LPC) release by platelets.LPC is released into extracellular vesicles (EVs) by activated platelets. This LPC might activate platelets in an autocrine/paracrine manner through the G2A receptor. LPC was also found to accumulate in unstable areas of atherosclerotic plaques.

might not only have a function as a biomarker but might also play an active role in the development of cardiovascular disease, potentially by mediating autocrine platelet activation and by being involved in the still poorly known process of plaque destabilization (-Fig. 1). However, some open questions remain for possible future studies. For example, it is unclear to which extent the LPC that was measured in mouse plasma is associated to platelet microvesicles. Likewise, although the authors convincingly showed that LPC is enriched in unstable plaques both from mice and humans, the origin of this LPC remains to be pinpointed exactly. Similar counts for its mechanistic relevance for plaque destabilization.

Taken together, the authors have demonstrated that LPC is a novel bioactive lipid mediator of platelet microvesicle activity with potential to become the grease for the progression of cardiovascular disease.

\section{Funding}

The author received funding from the Landsteiner Foundation for Blood Transfusion Research (LSBR Nr. 1638) and the European Union's Horizon 2020 research and innovation program under the Marie Skłodowska-Curie grant agreement No. 722609.

Conflict of Interest

None declared.

\section{References}

1 Diehl P, Peter K. Lysophosphatidylcholine is a major component of platelet microvesicles promoting platelet activation and report- ing atherosclerotic plaque instability. Thromb Haemost 2019;119 (08):1295-1310

2 Ridger VC, Boulanger CM, Angelillo-Scherrer A, et al; Position Paper of the European Society of Cardiology (ESC) Working Group on Atherosclerosis and Vascular Biology. Microvesicles in vascular homeostasis and diseases. Thromb Haemost 2017; 117(07):1296-1316

3 Dickhout A, Koenen RR. Extracellular vesicles as biomarkers in cardiovascular disease; chances and risks. Front Cardiovasc Med 2018;5:113

4 Valadi H, Ekström K, Bossios A, Sjöstrand M, Lee JJ, Lötvall JO. Exosome-mediated transfer of mRNAs and microRNAs is a novel mechanism of genetic exchange between cells. Nat Cell Biol 2007; 9(06):654-659

5 Chiva-Blanch G, Crespo J, Suades R, et al. CD142+/CD61+, CD146+ and CD45+ microparticles predict cardiovascular events in high risk patients following a Mediterranean diet supplemented with nuts. Thromb Haemost 2016;116(01):103-114

6 Santilli F, Marchisio M, Lanuti P, Boccatonda A, Miscia S, Davì G. Microparticles as new markers of cardiovascular risk in diabetes and beyond. Thromb Haemost 2016;116(02):220-234

7 Christersson C, Thulin Å, Siegbahn A. Microparticles during longterm follow-up after acute myocardial infarction. Association to atherosclerotic burden and risk of cardiovascular events. Thromb Haemost 2017;117(08):1571-1581

8 Koenen RR. The prowess of platelets in immunity and inflammation. Thromb Haemost 2016;116(04):605-612

9 Reddel CJ, Pennings GJ, Curnow JL, Chen VM, Kritharides L. Procoagulant effects of low-level platelet activation and its inhibition by colchicine. Thromb Haemost 2018;118(04): 723-733

10 Suades R, Padró T, Vilahur G, Badimon L. Circulating and platelet-derived microparticles in human blood enhance thrombosis on atherosclerotic plaques. Thromb Haemost 2012;108(06):1208-1219 
11 Barry OP, Praticò D, Savani RC, FitzGerald GA. Modulation of monocyte-endothelial cell interactions by platelet microparticles. J Clin Invest 1998;102(01):136-144

12 Vasina EM, Cauwenberghs S, Feijge MAH, Heemskerk JW, Weber C, Koenen RR. Microparticles from apoptotic platelets promote resident macrophage differentiation. Cell Death Dis 2011;2:e211

13 Sadallah S, Eken C, Martin PJ, Schifferli JA. Microparticles (ectosomes) shed by stored human platelets downregulate macrophages and modify the development of dendritic cells. J Immunol 2011;186 (11):6543-6552

14 Laffont B, Corduan A, Rousseau M, et al. Platelet microparticles reprogram macrophage gene expression and function. Thromb Haemost 2016;115(02):311-323

15 Vajen T, Benedikter BJ, Heinzmann ACA, et al. Platelet extracellular vesicles induce a pro-inflammatory smooth muscle cell phenotype. J Extracell Vesicles 2017;6(01):1322454 